\title{
The Molecular Basis of Mechanosensory Transduction
}

\author{
Kara L. Marshall ${ }^{1}$ and Ellen A. Lumpkin ${ }^{2, *}$ \\ ${ }^{1}$ Integrated Graduate Program in Cellular, Molecular, Structural and Genetic Studies, Columbia \\ University College of Physicians \& Surgeons, New York, NY 10032 \\ ${ }^{2}$ Departments of Dermatology and Physiology and Cellular Biophysics, Columbia University \\ College of Physicians \& Surgeons, New York, NY 10032
}

\begin{abstract}
Multiple senses including hearing, touch, and osmotic regulation, require the ability to convert force into an electrical signal: a process called mechanotransduction. Mechanotransduction occurs through specialized proteins that open an ion channel pore in response to a mechanical stimulus. Many of these proteins remain unidentified in vertebrates, but known mechanotransduction channels in lower organisms provide clues into their identity and mechanism. Bacteria, fruit flies, and nematodes have all been used to elucidate the molecules necessary for force transduction. This chapter discusses many different mechanical senses and takes an evolutionary approach to review the proteins responsible for mechanotransduction in various biological kingdoms.
\end{abstract}

\section{Introduction}

Behind the development of all senses is an organism's need to sample the surrounding world and extract information relevant for survival. Our bodies are bombarded by thermal changes, light, and chemicals, each of which has devoted encoding mechanisms; however, mechanisms that encode mechanical stimuli are perhaps the most commonly represented in the mammalian repertoire of senses.

Mechanosensation is necessary for mammals to perceive signals from the external world through touch and hearing, to monitor our internal states through sensing flow, osmotic pressure and blood pressure, and to perceive the relationship of our bodies to the external world through balance and proprioception. Though these senses seem quite disparate, they each encode a physical measurement of force. Advanced mechanosensory systems are not just present in mammals. All living things require some form of mechanosensation to survive- every cell responds to osmotic pressure and even single cell organisms react to touch. The ubiquitous presence of mechanotransduction suggests that it was one of the first senses to evolve. Although there is some evidence for convergent evolution, the majority of mechanisms for mechanotransduction are thought to have arisen divergently ${ }^{1}$. Microbial organisms developed the earliest mechanotransduction mechanisms to protect against cell rupture from osmotic shock. This basic mechanism is the evolutionary beginning of what sprouted into the vast array of mechanical senses present in more complex organisms.

\footnotetext{
*To whom correspondence should be addressed at eal2166@columbia.edu.
} 
Progress in elucidating fundamental mechanisms of mechanotransduction has come from exploring these processes in simple organisms. Despite their importance and prevalence, the mechanical senses remain the least understood in mammals. This chapter will discuss the development of mechanotransduction throughout the phylogenetic tree and the molecular mechanisms that underlie force sensing.

\section{Force Transduction}

The process of transforming a stimulus into a cellular signal is called transduction. In the nervous system, sensory transduction culminates in change of the electrical potential of a neuron This is accomplished by proteins in the membrane called ion channels, which are gated pores that allow the exchange of ions across the cell membrane. Sensory systems use specialized transduction molecules to convert stimulus energy into appropriate cellular signals. For example, the transduction molecule for vision (light) is rhodopsin, which changes conformation upon exposure to a photon. This begins a G-protein-coupled receptor signaling cascade that culminates in the gating of an ion channel. Although G-protein signaling is a commonly utilized mechanism for signal transduction, in mechanosensory systems, stimuli induce electrical activity by directly gating transduction channels.

Two primary models for the gating of mechanotransduction channels have been proposed (Fig. 1). The first model predicts that mechanotransduction channels are directly gated by force applied to the lipid membrane (Fig. 1a). This is the mechanism used by all known prokaryotic mechanosensitive ion channels. The second model posits that efficient chanel gating requires a tether to the cytoskeleton or extracellular matrix (Fig. 1b). Force placed on this tether creates a tension that opens the ion channel. In the case of the mammalian hair cell, this mechanism seems to be most likely.

Although it is possible that gating occurs indirectly through a secondary messenger cascade, there is no experimental evidence supporting this in any known mechanotransduction channel. Corey and Hudspeth used vestibular hair cells to demonstrate that the time between mechanical stimulation and an electrical response is $\sim 40 \mu s^{2}$. By contrast, the latency for phototransduction is tens of milliseconds due to signaling intermediates. The speed of transduction indicates that, at least for hair cells, force transduction molecules are forcegated ion channels.

\section{Prokaryotic mechanotransduction}

One can imagine a primordial world full of water, solutes and the first life: a unicellular prokaryote. With the development of a plasma membrane encapsulating this first organism, there came an inherent delicacy. The semi-permeability of the plasma membrane is essential for a cell's ability to sense and respond to its external environment, but requires tight control of the cell's internal environment and all of the solutes regulating its metabolic functions. The internal environment of this plasma membrane bag is dominated by water, and thus is subject to the laws of osmosis. Water will flow through the plasma membrane into a cell from a hypotonic environment, and out of a cell in a hypertonic one, so there must be a mechanism in place to preserve cell volume and membrane integrity. This is why 
osmotransduction is the most evolutionarily ancient mechanical sense and the primary mode of mechanotransduction in the prokaryotic kingdoms Bacteria and Archaea.

\section{Bacteria}

When exposed to an hypotonic external stimulus such as rain, bacteria require a mechanism to protect themselves from lysing. Osmotic pressure develops inside a cell when its external solution is diluted, causing the cell membrane to swell outward. In the 1950's, scientists observed that Escherichia coli eject intracellular solutes to compensate for a hypotonic challenge. Nearly fifty years later, the osmosensitive channels MscL, MscS, and MscM (mechanosensitive channels of large, small, and mini conductance) were discovered to be the emergency release valves that mediate this response ${ }^{3,4}$. They are named for the size of their conductances, $\sim 3 \mathrm{nS}, \sim 1 \mathrm{nS}$, and $\sim 0.3 \mathrm{nS}$, respectively, and require increasingly more membrane tension to open with increasing channel conductance. The pores of these channels are mostly large and non-selective to allow passage of a wide variety of solutes. Initially, Msc channels were studied using the patch clamp technique, as applying suction to the membrane caused large current steps. The same results were subsequently obtained by diluting the external solution, which deforms the plasma membrane due to increased intracellular osmotic pressure. ${ }^{5}$. Membrane deformation opens the Msc channel pore through which solutes are released, and membrane tension is then relieved ${ }^{3}$.

Martinac and colleagues expressed MscL in liposomes to examine the gating mechanism, and found that the channel was opened by changes in lipid tension alone. When lysophosphatidylcholine (LPC) was added symmetrically to liposomes reconstituted with MscL, the channel remained closed. However, with asymmetric addition of LPC, there is an asymmetric change in pressure across the bilayer that parallels the change in membrane curvature achieved with suction or cell swelling. This change in the lipid pressure profile was enough to open MscL in the absence of any suction, confirming the bilayer tension model in Fig $1^{6,7}$. The crystallization of MscL gave insight into the structural changes involved in the physical mechanism of pore opening: it is clear that the subunits twist against one another and flatten when tension is applied to the membrane, opening like the iris of a camera ${ }^{3}$.

Mutational studies provide compelling evidence that these channels are playing a protective, osmoregulatory role in bacteria. Booth and colleagues created E. coli with dysfunctional mutant $\mathrm{MscS}$ or MscL, as well as a double mutant ${ }^{8}$. Each single mutant was able to compensate for increased turgor during a hypo-osmotic challenge, but the double mutant $E$. coli lysed even with mild hypotonic shock. This showed that the smallest mechanotransduction channel, MscM, is not sufficient to protect bacteria from hypotonic environments.

The discovery of these channels in bacteria launched searches for similar genes in other organisms, the most related of which are prokaryotic members of the kingdom Archaea. 


\section{Archaea}

Osmoregulatory channels allow bacteria to survive in a broad range of osmotic environments, but members of a separate prokaryotic domain, Archaea, have adapted to the most extreme habitats on earth. These unicellular organisms are known to live in hypersaline marine environments such as the dead sea (halophiles), deep sea volcanic vents (methanogens), and acidic hot springs (thermoacidophiles). Mechanotransduction channels have been discovered in all three types of Archaea. The genome of Methanococcus jannaschii was the first Archaeon to be sequenced, and provided an opportunity to look for MS channel homologs. When probing with the most highly conserved region of the MscL protein, transmembrane domain 1 (TM1), scientists identified a residue with 38.5\% homology ${ }^{9}$. Conserved residues in the matching sequence were shown to be functionally important in bacterial MscL, and this new Archaeic protein was named MscMJ. Eventually two MS channels, MscMJ and MscMJLR, were identified in this species. These channels biophysically correspond most closely with MscS and MscL of E. coli, respectively. Interestingly, these sequence probe studies led to the discovery of another homolog to an $E$. coli protein named YggB, whose function was unknown at the time. YggB was later identified as the bacterial MS channel $\mathrm{MscS}^{8}$. The halophile Haloferax volcanii contains two distinct MS channels, MscA1 and MscA2, and the thermoacidophile Thermoplasma acidophilum contains MscTA ${ }^{9}$.

Like bacterial mechanotransduction channels, all discovered Archaeal channels are gated through lipid bilayer force, and open a large, mostly non-selective pore. These common channel properties fulfill the essential requirements for small, unicellular osmosensors, whose primary purpose is to rapidly compensate for osmotic changes in the environment. The sequence similarities of mechanosensitive channels in both Bacteria and Archaea point to a common MscL-like ancestor that likely arose before the two domains separated during evolution.

\section{Eukaryotic mechanotransduction}

In-depth studies of MscL channels provided a promising starting point for finding mechanically sensitive homologues in eukaryotes. The function of mechanotransduction channels has expanded to immensely diverse systems within the eukaryotic kingdoms, but osmosensation is still prevalent and integral to eukaryotic functioning. In addition, eukaryotes can react to varied stimuli like touch, sound and gravity. Plants contain channels similar to bacterial MscS, although no eukaryotic MscL-like channels have been found in the sequenced genome. These channels are proposed to confer osmoregulatory function and touch-sensitivity to plants ${ }^{10}$.

\section{Plants}

Plants have learned to thrive in myriad forms, but do so while being rooted in a single location, unable to escape from danger. To cope with this static lifestyle, they have developed defense mechanisms to protect themselves from assaults by wind, rain, and predators. Plant shoots and roots have also developed the ability to detect and avoid barriers encountered during growth, a response called thigmomorphogenesis. 
All plants respond to touch, but can do so in dramatically different ways. Specialized sensory cells allow some plants to react to touch on a very quick timescale with less than one second reaction time. Touching a Mimosa pudica will result in rapid leaf folding, as will brushing the trigger hairs of a Venus Fly Trap. While for most plants this response is protective, some plants have co-opted this ability for predatory use. Even in the absence of specialized cells that result in fast touch responses, plants respond to touch by changing gene expression and growth patterns ${ }^{11}$.

The model plant Arabidopsis thaliana has ten MscS-like (MSL 1-10) proteins with some sequence homology to bacterial and archaeal mechanotransduction channels. MSL 2 and 3 have been identified as necessary for proper organelle morphology and volume regulation, which are both dependent on osmotic sensation and control. To demonstrate the osmotransduction capacity of MSL3, Haswell and Meyerowitz expressed this channel in mutant E. coli that lacked all native osmoregulatory Msc channels. Normally these cells lyse in response to a hypotonic solution, but expression of plant MSL3 was able to rescue the bacterial cultures, demonstrating this channel is capable of functioning as an osmosensor ${ }^{12}$. MSL9 and MSL10 have also been identified as stretch-activated channels, and if all of the expressed MSL channels are removed from the plant root protoplasts, they lose all mechanically induced electrical activity ${ }^{10}$.

Strangely, mutants lacking all of these genes are phenotypically indistinguishable from wildtype, therefore, our understanding of their physiological role is still limited. Although MSL conductances are considerably smaller than those of MscS channels in bacteria, they are among the largest channel conductances ever recorded in plants. The relatively high conductances indicate these channels could function similarly to bacterial Msc channels, as this allows for a rapid membrane depolarization to correct for increased intracellular pressure.

\section{Caenorhabditis elegans and the molecular basis for touch}

When considering the sense of touch, the first thought that arises is distinctly mammalian: the feel of another's skin or the texture of an object in hand. This view neglects the widespread presence and importance of touch sensation in other organisms. Plants were the first unlikely touch-sensitive organisms discussed, but the eukaryote that has helped us understand the most about touch is a worm, Caenorhabditis elegans.

This small soil nematode is an ideal candidate for elucidating the molecular mechanism of touch because the genetics and development of all 302 neurons is well understood. $C$. elegans lives underground, and therefore must rely heavily on its mechanical senses to interact with its environment. The nematodes exhibit several stereotyped touch-response behaviors, many of which rely on different neurons that have been identified with laser ablation and genetic studies. The nose touch response, in which C. elegans reverses direction upon contacting an object in its path, is mediated by mechanosensory neurons ASH, FLP, and OLQ. Two ion channels belonging to the transient receptor potential (TRP) family have been shown to be responsible for mediating this behavior: OSM-9 and OCR-2 ${ }^{13,14}$. These same channels are also required for avoidance of hypertonic stimuli, which indicate a relationship between channels in Bacteria and Archaea. 
Although first discovered in the fruit fly (Drosophila melanogaster) retina, TRP channels have been widely implicated in mechanotransduction. They are widely expressed throughout eukaryotic phyla, and have provided numerous leads in the hunt to find mechanotransduction channels in various organisms. For example, yeast (Saccharomyces cerevisiae) requires TRPY1 to have proper fluid regulation responses to hypertonic stimuli ${ }^{15}$.

Another well understood touch response in C. elegans is the body touch response, in which the nematode changes directions when gently brushed with an eyelash along its body wall. Six specific neurons mediate this response, and these have distinct structural features that distinguish them from other body neurons. Gentle touch neurons are surrounded by a specialized extracellular matrix called the mantle, and they are filled with unusual 15protofilament microtubules ${ }^{16}$. These features suggest a tethered gating mechanism as they are unique to touch-sensitive neurons. Pioneering genetic screens by Chalfie and colleagues $^{17,18}$, identified 16 mec genes (mechanosensory abnormal) specifically required for the gentle touch response. Out of these 16 genes, two encode ion channel subunits (mec-4 and mec-10). These channels are a part of the DEG/ENaC superfamily of ion channels, which includes many ion channels expressed in mammals.

Along with the TRP family, DEG/ENaC channels are now primary candidates for investigating mechanotransduction. Mutant animals with non-functioning mec-4 and 10 have morphologically and developmentally normal touch neurons, but do not respond to mechanical stimuli. Nevertheless, when these two channel genes along with accessory subunits encoded by mec- 2 and mec- 6 are expressed heterologously in xenopus oocytes, they cannot be gated by mechanical stimuli ${ }^{19}$. The common hypothesis to explain this phenomenon is that proper function of the proteins requires expression of the other mec genes, many of which encode structural proteins that could help gate the channel or increase functional activity. Extracellular matrix components encoded by mec-1, mec-5, and mec-9 aide in the proper localization of the Mec complex, for example ${ }^{20}$. Although some studies supporting a model in which several mec proteins provide structural support that contributes to channel gating ${ }^{21}$, recent evidence shows otherwise. Specialized microtubules encoded by mec-7 and mec-12 are required for proper touch transduction, but do not interact with the mechanotransduction complex.

Defects in other mechanical senses in C. elegans have also pinpointed putative mechanosensory channels. Loss of unc-8, which encodes a Deg/ENaC subunit similar to MEC-4 and MEC-10, causes an uncoordinated phenotype in worms, suggesting a possible role in proprioceptive coordination of muscle movement ${ }^{22}$.

Identifying important genes that encode molecules necessary for touch was key groundwork for furthering the study of mechanotranduction. The next area ripe with possibilities was a different model animal with powerful genetic accessibility, and more complex mechanical senses than C. elegans. 


\section{Drosophila Melanogaster}

Fruit flies may be pests in a home but they are a boon to scientists in the lab. They have been a valuable tool to parse out the functions of myriad genes, as they have a short generation time and numerous tools are available to manipulate their genome. Even as a simple model system, they have developed many mechanical senses that are essential for their survival. In fact, mechanosensory mutant flies are completely incapacitated and fail to thrive in a laboratory setting. Along with sensing touch, flies require hearing to detect mating songs, proprioception to coordinate six multi-jointed legs, and strain gauges on their wings to control their flight path.

Externally, flies sense touch through type I mechanosensory bristles and chordotonal organs that cover their body. Each mechanosensory organ is independently innervated by single neuron with a ciliary structure extending into the bristle base (Fig. 2a). Bristles act as levers that transmit force when they are moved and, at their base, they may be able to detect movements as small as $1-2 \mathrm{~nm}^{23}$. When a bristle is bent, the proximal end compresses the dendritic shaft surrounding the cilia against the outer segment membrane, which contains tubular bundles (Fig. 2). Without these tubular bundles, mechanotransduction is lost, so the compression of the ciliary membrane against this structure is the putative gating stimulus ${ }^{24}$. Alternatively, this compression may be converted into a shear or tension force to gate mechanotransduction channels. Once the channels are opened, electrophysiological recordings reveal an inward $\mathrm{K}^{+}$current upon displacement of the bristle tip. As replacing the pipette solution with $\mathrm{Na}^{+}$does not affect the current, transduction molecules are nonselective cation channels.

Chordotonal organs are mechanosensory units that have no external process. They are attached to the cuticle, and the neuron is secured basally and apically, thus acting essentially as a stretch organ. An array of chordotonal organs, named Johnston's organ, comprises the fly's antennal ear. Although bristles rely on compression to gate channels, shear forces pulling on a dendritic cap connected to neuronal cilia is the stimulus in chordotonal organs (Fig. 2a).

The first putative D. melanogaster mechanotransduction channel to be identified, NOMPC, is a member of the TRPN branch of the TRP channel family. Mutation in the nompC gene eliminates most of bristle's mechanotransduction current, and the protein that it encodes is a fitting candidate for a mechanotransduction molecule. Despite seemingly different stimuli, the nompC mutants that showed no bristle potential also had greatly reduced receptor potentials for antennal sound responses ${ }^{25}$, which implicates NOMPC in chordotonal transduction and hearing.

Although its subcellular location has not been confirmed in D. melanogaster, the C. elegans homolog TRP-4 localizes to ciliary outer segments of specific neurons that contain tubular bundles similar to those in fly bristle sensory organs, making it a mechanotransduction molecule candidate ${ }^{26}$. Researchers have also been interested in another potentially important feature of NOMPC channels: out of the entire TRP superfamily, they contain the largest number (29) of ankyrin repeats. 
Ankyrins are structural proteins that commonly mediate protein-protein interactions. Many models of mechanical gating include a spring-like component in series with a tether, and the ankyrin helical structure makes it an attractive possibility for an intracellular gating spring ${ }^{27}$. These aspects together fit with the possibility that NOMPC is a mechanotransduction channel for fruit fly bristle sensation. A homolog of NOMPC, TRPN1, has also been shown to contribute to mechanotransduction in zebrafish hair cells ${ }^{28}$.

Other members of the TRP family, TRPV channels, also seem to be required for transduction in chordotonal organs. Mutations in nanchung (nan) and inactive (iav) resulted in deaf flies without sound-evoked potentials ${ }^{29,30}$. Both of these proteins are found specifically in the mechanosensory cilia of chordotonal organs. Moreover, each requires expression of the other for proper subcellular localization, suggesting that they form a heteromeric channel. This is comparable to the relationship of functional interdependence seen with closely related $C$. elegans channels OCR-2 and OSM- ${ }^{14}$. The exact subcellular location of the IAV protein is notable, as it is restricted to the proximal end of the neuronal cilia, which would require it to be gated by membrane tension rather than direct pulling by the dendritic $\operatorname{cap}^{29}$ (Fig. 2). The mechanosensitivity of these channels has been confirmed in heterologous expression systems, as both NAN and IAV are independently opened by hypotonic stimuli. The currents produced in this setting are very slow, and thus do not reflect the true speed of transduction in vivo. It is possible that proper transduction only occurs with the presence of either both channels together or another accessory molecule.

The fly's primitive ear possesses intriguing parallels with the vertebrate ear. It consists of a sail-like flagellum called the arista connected to the funiculus. When moved by mechanical force, the arista rotates the third antennal segment relative to the second, thus deforming the cuticle between the joint of the two segments, where the sensory units attach ${ }^{24}$. The mammalian cochlea is structurally and functionally quite different from the fly antennal auditory organ, and this has led to a common belief that the two arose separately during evolution. Surprisingly, genetic evidence has now revealed that many key genes required for proper development of the antennal auditory organ are also required for normal development or function of the vertebrate ear ${ }^{31}$. The proneural gene atonal is essential for differentiation of all chordotonal organs. Mammalian knockouts of the atonal homolog gene (atohl) lack both the mechanosensory hair cells of the ear ${ }^{32}$ and mechanosensitive Merkel cells of the $\operatorname{skin}^{31,33}$. Additionally, the vertebrate cochlea has the ability to amplify and augment sounds so that they respond non-linearly to acoustic energy. The mechanism varies in mammals and tetrapods, but is accomplished by contraction of the hair cell itself or through movement of apical hair-cell bundles. Gopfert and Robert found that auditory neurons in the fly show a remarkably similar nonlinear tuning, thus mirroring both the actuating and transducing roles of the vertebrate cochlea ${ }^{34}$.

This small organ has provided much insight into mechanisms of hearing, as well as other mechanical senses. Aside from hearing courtship songs and other relevant stimuli, the Johnston's organ is also responsible for Drosophila's ability to sense gravity and wind ${ }^{35,36}$. The third antennal subunit is deformed by gravity irrespective of head orientation, thereby providing the "gyroscope" function needed for proper flight ${ }^{35}$. 
In addition to ciliated mechanosensory neurons, non-ciliated neurons that innervate the fly's body wall have been proposed to function as proprioceptors and nociceptors, or pain neurons. Tracey and colleagues found that larvae have very different behavioral responses to a harsh touch or a painfully hot $\left(>38^{\circ}\right)$ probe than they do to a gentle touch. The distinct writhing escape response indicated that there is a pain-specific sensory pathway, and three painless mutants lacked this response ${ }^{37}$. All three painless lines had mutations in a TRPA channel subunit, which was later found to be required for thermal nociception in adult flies and avoidance of isothiocyanates ${ }^{38}$. The closely related channel TRPA1 is found in mammals, where it is also essential for isothiocyanate sensitivity. Thus, this polymodal channel senses mechanical, thermal, and chemical stimuli. This pathway is completely distinct from chordotonal transduction, as atonal mutants (which lack chordotonal organs) retain the writhing response, and painless mutants have no deficit in chordotonal transduction.

These mechanosensory neurons in Drosophila have been immensely powerful in teaching us about the mechanisms underlying various representations of mechanical senses. These sensory abilities only become more fascinating, complex and varied when investigating higher branches of the phylogenetic tree.

\section{Vertebrate Mechanotransduction}

Of the many specialized mechanosensory systems found across phyla, vertebrates have developed the most elaborate. The following section will focus primarily on mammalian mechanosensory systems.

Several attributes of mammalian mechanotransduction channels make them difficult to identify. Mechanosensory cells or receptor endings are few in number and are not readily separated from surrounding cells. This is most evident in the cochlea, which only has about 15,000 hair cells, making biochemical assays difficult. Additionally, these specialized sensors are estimated to have very few transduction channels. For example, each hair cell is estimated to only have 50-100 transduction channels. Lastly, it is possible that mammalian mechanotransduction channels are multiprotein complexes, unlike experimentally tractable bacterial channels that are encoded by single genes.

These hurdles in uncovering mammalian mechanotransduction channels are slowly being overcome with new technology and creative scientific minds, but the molecular mechanisms behind mammalian touch reception still remain a mystery.

\section{The somatosensory system - touch, nociception and prioprioception}

The skin acts as our first line of defense, sensing both damaging and pleasant stimuli. Sensory neurons innervating the skin are responsible for conveying thermal, chemical and mechanical information. It is clear that some of these stimuli activate separate transduction mechanisms, whereas other proteins can be activated by more than one stimulus. TRPV1 (transient receptor potential vanilloid 1), for instance, is activated both by heat and chemicals. 
The skin is densely innervated by mechanosensory nerve endings, such as lanceolate endings around hair follicles, Pacinian corpuscles, Meissner's corpuscles, and Merkel cellneurite complexes. As yet, the molecules that confer force sensitivity to any of these sensory endings are largely unknown.

One promising avenue to identify candidate transduction molecules is to analyze mammalian homologs of C. elegans Mec genes. For example, knockout mice lacking a MEC-2 related protein, stomatin-like protein 3 (SLP3), show deficits in discriminating texture and in electrophysiological recordings from touch-sensitive afferent nerves. This indicates SLP3 is essential for mechanotransduction in some cutaneous sensory afferents ${ }^{39}$.

Similarly, several studies implicate DEG/ENaC ion channels in mammalian somatosensory mechanotransduction. Mice lacking acid-sensing ion channels (ASIC) 2 and 3 resulted in either no touch deficits ${ }^{40}$, or moderate ones ${ }^{41,42}$; however, the mechanical threshold for these touch-sensitive neurons are not changed. A dominant-negative mutant of ASIC3 actually increased the sensitivity of fibers to mechanical and chemical stimuli ${ }^{43}$. These data suggest that mammalian ASIC channels shape the output of touch-sensitive afferents, rather than serve as mechanotransduction channels in these neurons.

Another useful approach for analyzing mechanotransduction mechanisms is to study sensory neurons in simplified, in vitro preparations. The cell bodies of somatosensory neurons cluster in dorsal root ganglia (DRG) and trigeminal ganglia (TG), which can be easily dissociated and cultured so that mechanically activated responses can be monitored with electrophysiology or calcium imaging. Dissociated DRG neurons possess a number of mechanically activated currents that may reflect bona fide mechanotransduction mechanisms. For example, subsets of DRG neurons can be activated by radial stretch ${ }^{44}$, hypotonic stimuli ${ }^{45}$, suction ${ }^{46}$ and direct touch ${ }^{47}$. Intriguingly, Lewin and colleagues recently reported that some mechanically evoked currents in DRG neurons require extracellular tethers 48 .

Another promising model system for analyzing touch transduction mechanisms lies in a specialized light-touch receptor called the Merkel cell-neurite complex. These touch receptors cluster close to the skin's surface, and each cluster is innervated by a single sensory afferent that branches multiple times, sending a single neurite to each Merkel cell. Merkel cells are thought to be required for essential tasks that rely upon fine tactile discrimination. The sensory afferent innervating the Merkel cell-neurite complex has unique electrophysiological signature, and abolishing these cells removes this neural light-touch signal $^{49}$. Touch researchers have pioneered methods to culture and enrich Merkel cells so that their mechanotransduction mechanisms can be directly assessed. In culture, Merkel cells are activated by hypotonic-evoked cell swelling and their response involves amilorideinsensitive, calcium-permeable ion channels ${ }^{50,51}$. To begin the search for the underlying mechanotransduction channels, Lumpkin and colleagues investigated the gene expression profile of Merkel cells. Interestingly, several TRP channels are expressed in Merkel cells, which provide a starting point for investigating putative mechanotransduction channels ${ }^{52}$. 
Along with the sense of touch, somatosensory neurons allow us to determine where our limbs are in space, by conveying joint angle, muscle tension and muscle length. These proprioceptive afferents include muscle spindle organs, which are responsible for conveying muscle length information. At the end of the muscle in the tendon are Golgi tendon organs, which interweave with collagen fibrils to convey information about muscle tension. Both of these afferent types provide feedback to the brain, allowing animals to protect their muscles from overstretching and to form a neural representation of their body position. Ion channels involved in these mechanisms are not known.

\section{The acoustico-lateralis system - hearing and balance}

The cochlea, an intricate organ responsible for sound-detection in mammals, is one of the most specialized and sensitive mechanisms developed to detect mechanical force. It sits encased in the skull, with a coil of three fluid-filled chambers. The center chamber contains a fluid called endolymph, which has a high concentration of potassium. Distributed along the partition of these chambers sits the Organ of Corti, which contains mechanically sensitive hair cells. Pressure from a sound wave deforms the tympanic membrane (eardrum), vibrating the small bones of the middle ear. The vibrations are transmitted to the oval window, which pushes waves of pressure into the fluid filled cochlea. Endolymph movements cause displacement of hair bundles, clusters of specialized microvilli called stereocilia that are the site of mechanotransduction.

Stereocilia are actin-filled processes arranged in a height-dependent manner, with rows cascading down from a single microtubule-filled kinocilium. Each level of stereocilia is connected to the one above it by a spring protein strand called the tip link, which is proposed to be in series with the gating spring that opens mechanotransduction channels when hair bundles move (FIG 2b). Although this intricate structure of the hair bundle is well understood, many of the molecular components responsible for mechanotransduction remain a mystery, however; protein components of the tip link were recently shown to be cadherein 23 and protocadherin $15^{53}$.

Hair cells of the cochlea share common morphology and mechanisms of action with hair cells of the vestibular system. The utricle, saccule and semicircular canals allow for the detection of gravity, and provide feedback for balance. Due to common morphology and function, it is possible that all hair cells in the inner ear will employ mechanotransduction channels encoded by the same genes

\section{Mammalian stretch-sensitive ion channels}

Although hair cells are proposed employ tethered transduction channels, stretch-sensitive ion channels have been found widely in mammalian cell types. In most cases the molecular identities of these channels is unclear; however, one class shares a notable structural feature with prokaryotic channels. MscL, MscS, and MscMJ share a cluster of charged residues in their C-terminus, and removal of this sequence from MscL abolishes the channel's mechanosensitivity of the channel ${ }^{54,55}$. A very similar sequence was found in the cterminus of the mammalian mechanically gated potassium channel TREK-1 ${ }^{9}$. TREK-1 is a stretch-sensitive two-pore domain potassium channel that opens preferentially to convex

Adv Exp Med Biol. Author manuscript; available in PMC 2014 June 17. 
stimuli and loses mechanosensitivity with loss of its C-terminus ${ }^{56}$. Evidence from knockout animals suggests that TREK-1 may be important for tuning the mechanosensitivity of nociceptors, as deletion of TREK-1 causes allodynia, an increase in sensitivity to nonpainful mechanical stimuli. Intriguingly, mice lacking TREK-1 show decreased sensitivity to mechanical and thermal hyperalgesia, which indicates this channel plays a role in sensitizing nociceptors ${ }^{57}$. Two related potassium channels TREK-2, and TRAAK, are also stretch-sensitive ${ }^{58}$.

Other senses in mammals that might require stretch-sensitive channels include baroreception, bladder osmosensation and bladder voiding. The TRP channel TRPV4 contributes to osmosensation in mammals, as mice lacking this channel are not able to regulate their osmotic equilibrium as efficiently as wild-type mice ${ }^{59}$. Additionally, TRPV4 contributes to mechanically evoked visceral pain ${ }^{60}$. This channel was also found to transduce mechanical shear stress in an ex vivo carotid artery preparation ${ }^{61}$. In TRPV4 knockout artery tissue, there was an absence of this stress response, indicating this channel could contribute to baroreception.

The myriad mechanical senses of mammals are excellent examples of the power of evolution to shape complex sensory responses. We communicate, navigate through space and control our internal states all through detecting forces inside and around us. The mystery of these abilities is slowly being uncovered, but mechanotransduction remains a ripe area for discovery.

\section{Acknowledgments}

The authors were supported by NIAMS grant AR051219 (to EAL), by the Rush and Helen Record Foundation (to KLM) and by NIGMS grant 2T32GM008507-16 to the Department of Neuroscience, Baylor College of Medicine. Portions of this chapter were prepared in the Department of Neuroscience, Baylor College of Medicine, Houston TX. We thank members of the Lumpkin laboratory for helpful discussions.

\section{References}

1. Martinac B. Mechanosensitive ion channels: molecules of mechanotransduction. J Cell Sci. 2004 May 15; 117(Pt 12):2449-60. [PubMed: 15159450]

2. Corey DP, Hudspeth AJ. Kinetics of the receptor current in bullfrog saccular hair cells. J Neurosci. 1983 May; 3(5):962-76. [PubMed: 6601694]

3. Kung C. A possible unifying principle for mechanosensation. Nature. 2005 Aug 4; 436(7051):64754. [PubMed: 16079835]

4. Berrier C, Besnard M, Ajouz B, Coulombe A, Ghazi A. Multiple mechanosensitive ion channels from Escherichia coli, activated at different thresholds of applied pressure. J Membr Biol. 1996 May; 151(2):175-87. [PubMed: 8661505]

5. Martinac B, Buechner M, Delcour AH, Adler J, Kung C. Pressure-sensitive ion channel in Escherichia coli. Proc Natl Acad Sci U S A. 1987 Apr; 84(8):2297-301. [PubMed: 2436228]

6. Perozo E, Cortes DM, Sompornpisut P, Kloda A, Martinac B. Open channel structure of MscL and the gating mechanism of mechanosensitive channels. Nature. 2002 Aug 29; 418(6901):942-8. [PubMed: 12198539]

7. Perozo E, Kloda A, Cortes DM, Martinac B. Physical principles underlying the transduction of bilayer deformation forces during mechanosensitive channel gating. Nat Struct Biol. 2002 Sep; 9(9): 696-703. [PubMed: 12172537]

8. Levina N, Totemeyer S, Stokes NR, Louis P, Jones MA, Booth IR. Protection of Escherichia coli cells against extreme turgor by activation of $\mathrm{MscS}$ and $\mathrm{MscL}$ mechanosensitive channels: 
identification of genes required for MscS activity. EMBO J. 1999 Apr 1; 18(7):1730-7. [PubMed: 10202137]

9. Kloda A, Martinac B. Mechanosensitive channels in archaea. Cell Biochem Biophys. 2001; 34(3): 349-81. [PubMed: 11898861]

10. Haswell ES, Peyronnet R, Barbier-Brygoo H, Meyerowitz EM, Frachisse JM. Two MscS homologs provide mechanosensitive channel activities in the Arabidopsis root. Curr Biol. 2008 May 20; 18(10):730-4. [PubMed: 18485707]

11. Braam J. In touch: plant responses to mechanical stimuli. New Phytol. 2005 Feb; 165(2):373-89. [PubMed: 15720650]

12. Haswell ES, Meyerowitz EM. MscS-like proteins control plastid size and shape in Arabidopsis thaliana. Curr Biol. 2006 Jan 10; 16(1):1-11. [PubMed: 16401419]

13. Xiao R, Xu XZ. Function and regulation of TRP family channels in C. elegans. Pflugers Arch. 2009 Sep; 458(5):851-60. [PubMed: 19421772]

14. Tobin D, Madsen D, Kahn-Kirby A, Peckol E, Moulder G, Barstead R, et al. Combinatorial expression of TRPV channel proteins defines their sensory functions and subcellular localization in C. elegans neurons. Neuron. 2002 Jul 18; 35(2):307-18. [PubMed: 12160748]

15. Su Z, Zhou X, Loukin SH, Haynes WJ, Saimi Y, Kung C. The use of yeast to understand TRPchannel mechanosensitivity. Pflugers Arch. 2009 Sep; 458(5):861-7. [PubMed: 19462180]

16. Tavernarakis N, Driscoll M. Mechanotransduction in Caenorhabditis elegans: the role of DEG/ ENaC ion channels. Cell Biochem Biophys. 2001; 35(1):1-18. [PubMed: 11898851]

17. Chalfie M, Sulston J. Developmental genetics of the mechanosensory neurons of Caenorhabditis elegans. Dev Biol. 1981 Mar; 82(2):358-70. [PubMed: 7227647]

18. Chalfie M, Au M. Genetic control of differentiation of the Caenorhabditis elegans touch receptor neurons. Science. 1989 Feb 24; 243(4894 Pt 1):1027-33. [PubMed: 2646709]

19. Brown AL, Liao Z, Goodman MB. MEC-2 and MEC-6 in the Caenorhabditis elegans sensory mechanotransduction complex: auxiliary subunits that enable channel activity. J Gen Physiol. 2008 Jun; 131(6):605-16. [PubMed: 18504316]

20. Goodman, MB. Worm Book. 2006.

21. Drew LJ, Wood JN. Worm sensation! Mol Pain. 2005; 1:8.

22. Tavernarakis N, Shreffler W, Wang S, Driscoll M. unc-8, a DEG/ENaC family member, encodes a subunit of a candidate mechanically gated channel that modulates $\mathrm{C}$. elegans locomotion. Neuron. 1997 Jan; 18(1):107-19. [PubMed: 9010209]

23. Walker RG, Willingham AT, Zuker CS. A Drosophila mechanosensory transduction channel. Science. 2000 Mar 24; 287(5461):2229-34. [PubMed: 10744543]

24. Kernan MJ. Mechanotransduction and auditory transduction in Drosophila. Pflugers Arch. 2007 Aug; 454(5):703-20. [PubMed: 17436012]

25. Eberl DF, Hardy RW, Kernan MJ. Genetically similar transduction mechanisms for touch and hearing in Drosophila. J Neurosci. 2000 Aug 15; 20(16):5981-8. [PubMed: 10934246]

26. Li W, Feng Z, Sternberg PW, Xu XZ. A C. elegans stretch receptor neuron revealed by a mechanosensitive TRP channel homologue. Nature. 2006 Mar 30; 440(7084):684-7. [PubMed: 16572173]

27. Howard J, Bechstedt S. Hypothesis: a helix of ankyrin repeats of the NOMPC-TRP ion channel is the gating spring of mechanoreceptors. Curr Biol. 2004 Mar 23; 14(6):R224-6. [PubMed: 15043829]

28. Sidi S, Friedrich RW, Nicolson T. NompC TRP channel required for vertebrate sensory hair cell mechanotransduction. Science. 2003 Jul 4; 301(5629):96-9. [PubMed: 12805553]

29. Gong Z, Son W, Chung YD, Kim J, Shin DW, McClung CA, et al. Two interdependent TRPV channel subunits, inactive and Nanchung, mediate hearing in Drosophila. J Neurosci. 2004 Oct 13; 24(41):9059-66. [PubMed: 15483124]

30. Kim J, Chung YD, Park DY, Choi S, Shin DW, Soh H, et al. A TRPV family ion channel required for hearing in Drosophila. Nature. 2003 Jul 3; 424(6944):81-4. [PubMed: 12819662]

31. Boekhoff-Falk G. Hearing in Drosophila: development of Johnston's organ and emerging parallels to vertebrate ear development. Dev Dyn. 2005 Mar; 232(3):550-8. [PubMed: 15704117] 
32. Bermingham NA, Hassan BA, Price SD, Vollrath MA, Ben-Arie N, Eatock RA, et al. Math1: an essential gene for the generation of inner ear hair cells. Science. 1999 Jun 11; 284(5421):1837-41. [PubMed: 10364557]

33. Lumpkin EA, Collisson T, Parab P, Omer-Abdalla A, Haeberle H, Chen P, et al. Math1-driven GFP expression in the developing nervous system of transgenic mice. Gene Expr Patterns. 2003 Aug; 3(4):389-95. [PubMed: 12915300]

34. Gopfert MC, Robert D. Motion generation by Drosophila mechanosensory neurons. Proc Natl Acad Sci U S A. 2003 Apr 29; 100(9):5514-9. [PubMed: 12642657]

35. Yorozu S, Wong A, Fischer BJ, Dankert H, Kernan MJ, Kamikouchi A, et al. Distinct sensory representations of wind and near-field sound in the Drosophila brain. Nature. 2009 Mar 12; 458(7235):201-5. [PubMed: 19279637]

36. Kamikouchi A, Inagaki HK, Effertz T, Hendrich O, Fiala A, Gopfert MC, et al. The neural basis of Drosophila gravity-sensing and hearing. Nature. 2009 Mar 12; 458(7235):165-71. [PubMed: 19279630]

37. Tracey WD Jr, Wilson RI, Laurent G, Benzer S. painless, a Drosophila gene essential for nociception. Cell. 2003 Apr 18; 113(2):261-73. [PubMed: 12705873]

38. Xu SY, Cang CL, Liu XF, Peng YQ, Ye YZ, Zhao ZQ, et al. Thermal nociception in adult Drosophila: behavioral characterization and the role of the painless gene. Genes Brain Behav. 2006 Nov; 5(8):602-13. [PubMed: 17081265]

39. Wetzel C, Hu J, Riethmacher D, Benckendorff A, Harder L, Eilers A, et al. A stomatin-domain protein essential for touch sensation in the mouse. Nature. 2007 Jan 11; 445(7124):206-9. [PubMed: 17167420]

40. Drew LJ, Rohrer DK, Price MP, Blaver KE, Cockayne DA, Cesare P, et al. Acid-sensing ion channels ASIC2 and ASIC3 do not contribute to mechanically activated currents in mammalian sensory neurones. J Physiol. 2004 May 1; 556(Pt 3):691-710. [PubMed: 14990679]

41. Price MP, Lewin GR, McIlwrath SL, Cheng C, Xie J, Heppenstall PA, et al. The mammalian sodium channel BNC1 is required for normal touch sensation. Nature. 2000 Oct 26; 407(6807): 1007-11. [PubMed: 11069180]

42. Price MP, McIlwrath SL, Xie J, Cheng C, Qiao J, Tarr DE, et al. The DRASIC cation channel contributes to the detection of cutaneous touch and acid stimuli in mice. Neuron. $2001 \mathrm{Dec} 20$; 32(6):1071-83. [PubMed: 11754838]

43. Mogil JS, Breese NM, Witty MF, Ritchie J, Rainville ML, Ase A, et al. Transgenic expression of a dominant-negative ASIC3 subunit leads to increased sensitivity to mechanical and inflammatory stimuli. J Neurosci. 2005 Oct 26; 25(43):9893-901. [PubMed: 16251436]

44. Bhattacharya MR, Bautista DM, Wu K, Haeberle H, Lumpkin EA, Julius D. Radial stretch reveals distinct populations of mechanosensitive mammalian somatosensory neurons. Proc Natl Acad Sci U S A. 2008 Dec 16; 105(50):20015-20. [PubMed: 19060212]

45. Viana F, de la Pena E, Pecson B, Schmidt RF, Belmonte C. Swelling-activated calcium signalling in cultured mouse primary sensory neurons. Eur J Neurosci. 2001 Feb; 13(4):722-34. [PubMed: 11207807]

46. Takahashi A, Gotoh H. Mechanosensitive whole-cell currents in cultured rat somatosensory neurons. Brain Res. 2000 Jun 30; 869(1-2):225-30. [PubMed: 10865079]

47. McCarter GC, Levine JD. Ionic basis of a mechanotransduction current in adult rat dorsal root ganglion neurons. Mol Pain. 2006; 2:28. [PubMed: 16923187]

48. Hu J, Chiang LY, Koch M, Lewin GR. Evidence for a protein tether involved in somatic touch. EMBO J. Feb 17; 29(4):855-67. [PubMed: 20075867]

49. Maricich SM, Wellnitz SA, Nelson AM, Lesniak DR, Gerling GJ, Lumpkin EA, et al. Merkel cells are essential for light-touch responses. Science. 2009 Jun 19; 324(5934):1580-2. [PubMed: 19541997]

50. Haeberle H, Bryan LA, Vadakkan TJ, Dickinson ME, Lumpkin EA. Swelling-activated Ca2+ channels trigger Ca2+ signals in Merkel cells. PLoS One. 2008; 3(3):e1750. [PubMed: 18454189]

51. Haeberle H, Lumpkin EA. Merkel Cells in Somatosensation. Chemosens Percept. 2008 Jun 1; 1(2): 110-8. [PubMed: 19834574] 
52. Haeberle H, Fujiwara M, Chuang J, Medina MM, Panditrao MV, Bechstedt S, et al. Molecular profiling reveals synaptic release machinery in Merkel cells. Proc Natl Acad Sci U S A. 2004 Oct 5; 101(40):14503-8. [PubMed: 15448211]

53. Kazmierczak P, Sakaguchi H, Tokita J, Wilson-Kubalek EM, Milligan RA, Muller U, et al. Cadherin 23 and protocadherin 15 interact to form tip-link filaments in sensory hair cells. Nature. 2007 Sep 6; 449(7158):87-91. [PubMed: 17805295]

54. Blount P, Sukharev SI, Schroeder MJ, Nagle SK, Kung C. Single residue substitutions that change the gating properties of a mechanosensitive channel in Escherichia coli. Proc Natl Acad Sci U S A. 1996 Oct 15; 93(21):11652-7. [PubMed: 8876191]

55. Hase CC, Le Dain AC, Martinac B. Molecular dissection of the large mechanosensitive ion channel (MscL) of E. coli: mutants with altered channel gating and pressure sensitivity. J Membr Biol. 1997 May 1; 157(1):17-25. [PubMed: 9141355]

56. Dedman A, Sharif-Naeini R, Folgering JH, Duprat F, Patel A, Honore E. The mechano-gated K(2P) channel TREK-1. Eur Biophys J. 2009 Mar; 38(3):293-303. [PubMed: 18369610]

57. Alloui A, Zimmermann K, Mamet J, Duprat F, Noel J, Chemin J, et al. TREK-1, a K+ channel involved in polymodal pain perception. EMBO J. 2006 Jun 7; 25(11):2368-76. [PubMed: 16675954]

58. Patel AJ, Lazdunski M, Honore E. Lipid and mechano-gated 2P domain K(+) channels. Curr Opin Cell Biol. 2001 Aug; 13(4):422-8. [PubMed: 11454447]

59. Liedtke W, Friedman JM. Abnormal osmotic regulation in trpv4-/- mice. Proc Natl Acad Sci U S A. 2003 Nov 11; 100(23):13698-703. [PubMed: 14581612]

60. Brierley SM, Page AJ, Hughes PA, Adam B, Liebregts T, Cooper NJ, et al. Selective role for TRPV4 ion channels in visceral sensory pathways. Gastroenterology. 2008 Jun; 134(7):2059-69. [PubMed: 18343379]

61. Hartmannsgruber V, Heyken WT, Kacik M, Kaistha A, Grgic I, Harteneck C, et al. Arterial response to shear stress critically depends on endothelial TRPV4 expression. PLoS One. 2007; 2(9):e827. [PubMed: 17786199] 


\section{A Stretch-activated}

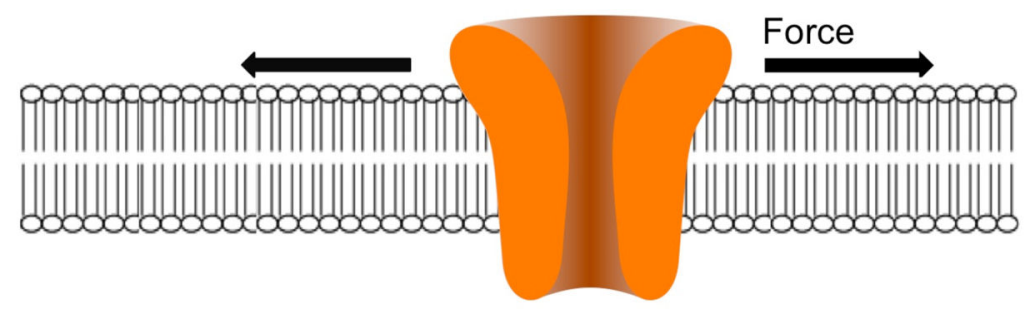

B Tethered
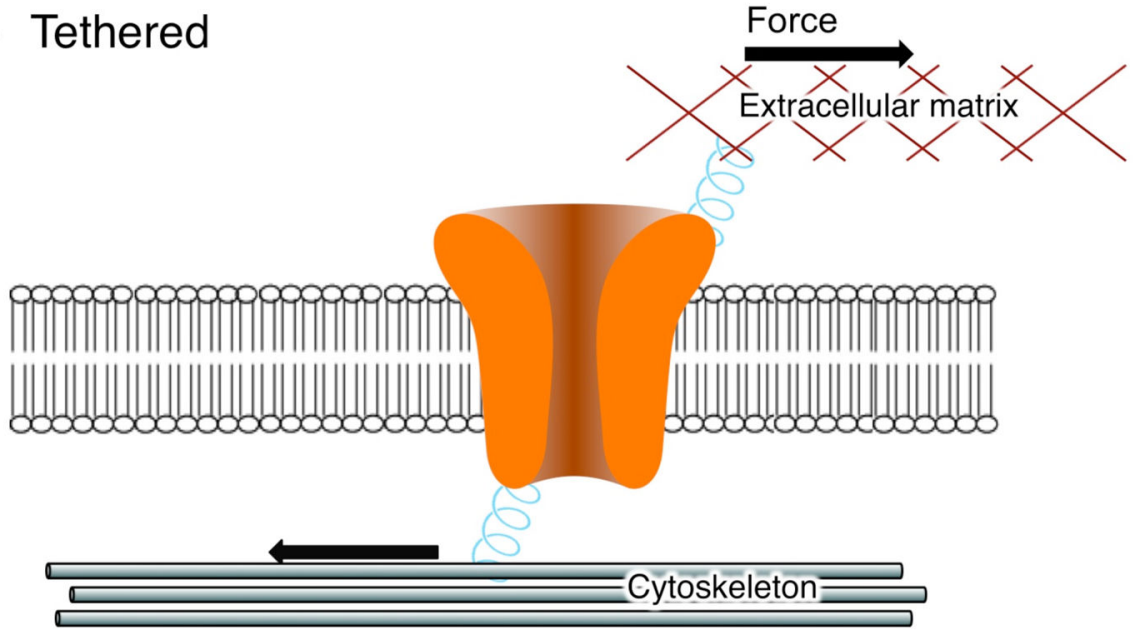

Fig. 1.

Two models of gating for mechanotransduction channels. A) Lipid bilayer tension is sufficient to cause channel opening, or B) mechanotransdcution channels require a tether connected to either extracellular matrix and/or cytoskeletal elements. 


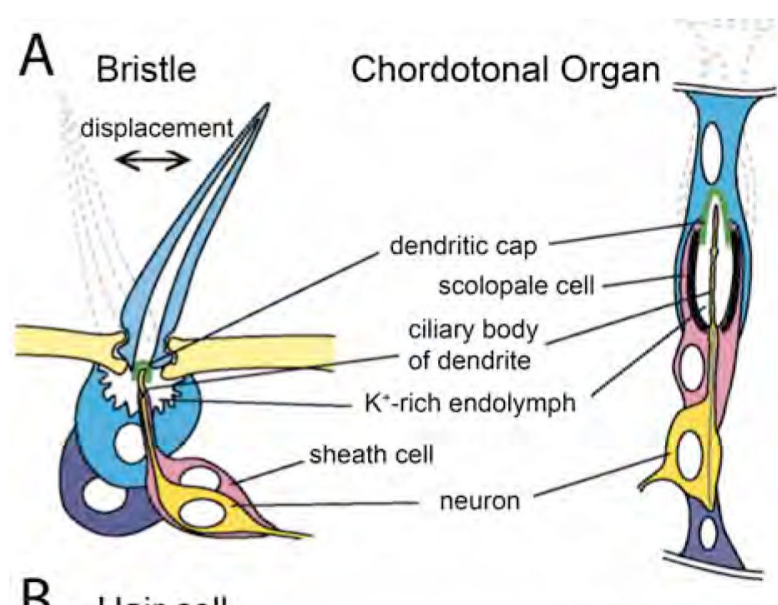

B Hair cell
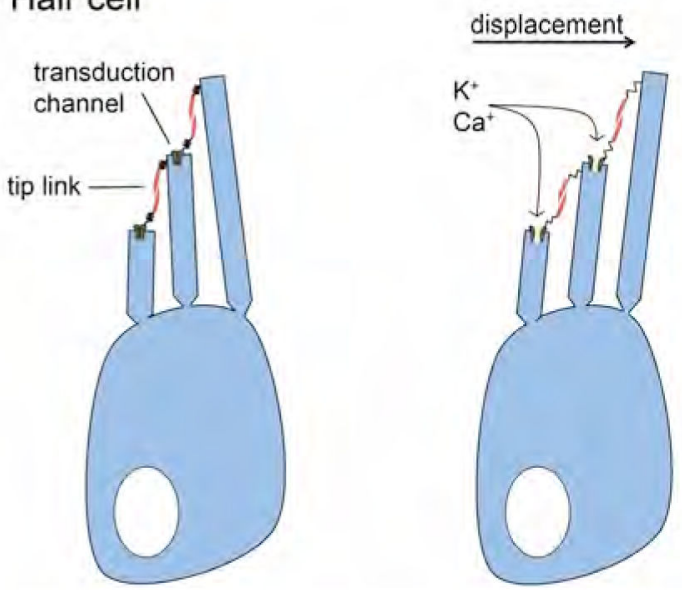

Fig. 2.

Mechanosensory cells. A) A depiction of two mechanosensory organs in D. melanogaster, and their proposed mechanisms of activation upon position change (shown by dotted outlines) as a result of a mechanical stimulus. In both organs, the neuronal dendrite is attached to a dendritic cap that is affected by stimulus-induced movement, activating mechanotransduction channels. Adapted with permission from: Jarmen A. N. et al. Human Molec. Genetics 2002; 11:1215-1218. B) A vertebrate hair cell before (left) and after (right) displacement by endolymph movement. The shear force pushing stereocilia creates tension in a spring-like element (as yet unidentified) that is proposed to be in series with the tip link. Increased tension results in transduction-channel opening. 